Self-diffusion and microscopic dynamics in a gold-silicon liquid investigated with quasielastic neutron scattering

Zach Evenson', Fan Yang, Giovanna G. Simeoni, and Andreas Meyer

Citation: Appl. Phys. Lett. 108, 121902 (2016); doi: 10.1063/1.4944814

View online: http://dx.doi.org/10.1063/1.4944814

View Table of Contents: http://aip.scitation.org/toc/apl/108/12

Published by the American Institute of Physics 


\title{
Self-diffusion and microscopic dynamics in a gold-silicon liquid investigated with quasielastic neutron scattering
}

\author{
Zach Evenson, ${ }^{1,2, a)}$ Fan Yang, ${ }^{2}$ Giovanna G. Simeoni, ${ }^{1}$ and Andreas Meyer $^{2}$ \\ ${ }^{1}$ Heinz Maier-Leibnitz Zentrum (MLZ) and Physik Department, Technische Universität München, \\ Lichtenbergstrasse 1, 85748 Garching, Germany \\ ${ }^{2}$ Institut für Materialphysik im Weltraum, Deutsches Zentrum für Luft- und Raumfahrt (DLR), 51170 Köln, \\ Germany
}

(Received 3 February 2016; accepted 11 March 2016; published online 22 March 2016)

\begin{abstract}
We use incoherent quasielastic neutron scattering to study the atomic dynamics of gold in a eutectic $\mathrm{Au}_{81} \mathrm{Si}_{19}$ melt. Despite the glass-forming nature of this system, the gold self-diffusivity displays an Arrhenius behavior with a low activation energy characteristic of simple liquids. At high temperatures, long-range transport of gold atoms is well described by hydrodynamic theory with a simple exponential decay of the self-correlation function. On cooling towards the melting temperature, structural relaxation crosses over to a highly stretched exponential behavior. This suggests the onset of a heterogeneous dynamics, even in the equilibrium melt, and is indicative of a very fragile liquid. @ 2016 AIP Publishing LLC. [http://dx.doi.org/10.1063/1.4944814]
\end{abstract}

Atomic transport in liquid metals controls many important physical processes such as diffusion, nucleation, crystallization, and glass-formation. ${ }^{1}$ Binary gold-silicon presents an exceptionally deep eutectic many hundreds of degrees below the melting temperatures of its constituent components ${ }^{2}$ and is a technologically interesting system regarding the growth of silicon nanowires, casting of carat gold alloys, and development of bulk metallic glasses. ${ }^{3}$ The unique physical properties of this system have promising technological implications, even though surprisingly little direct information is available about the microscopic dynamics.

Quasielastic neutron scattering (QENS) is a highly versatile technique for studying atomic transport in metallic melts and allows for self-diffusion coefficients to be measured in-situ with a high degree of accuracy. ${ }^{4}$ Here, we present results of a QENS investigation on the eutectic melt $\mathrm{Au}_{81} \mathrm{Si}_{19}$. Despite the large neutron absorption cross-section of gold ( $\sigma_{\mathrm{abs}}=384 \mathrm{~b}$ at $7 \AA$ incident wavelength), we, nevertheless, observe a broad quasielastic signal at small momentum transfer, which is a signature of the atomic diffusion of gold.

QENS was carried out in the equilibrium melt of $\mathrm{Au}_{81} \mathrm{Si}_{19}$ on the time-of-flight spectrometer TOFTOF ${ }^{5}$ at the Heinz Maier-Leibnitz neutron source (FRM II) in Munich. Sample plates of $1 \mathrm{~mm}$ thickness were placed inside of a rectangular niobium container with a total wall thickness of $2 \mathrm{~mm}$, which was then hermetically sealed under highvacuum conditions using electron beam welding. The eutectic temperature $T_{m}$ of the alloy was measured using differential scanning calorimetry as $648 \mathrm{~K}$, in good agreement with the published phase diagram. ${ }^{2}$ The measurements were carried out with the sample oriented in a reflection geometry, and spectra were collected on heating at five temperatures above $T_{m}(T=1163,1103,1007,862$, and $713 \mathrm{~K})$. A separate measurement of vanadium at room temperature gave the instrument energy resolution function.

${ }^{\text {a)} E l e c t r o n i c ~ m a i l: ~ Z a c h a r y . E v e n s o n @ f r m 2 . t u m . d e ~}$
An incident neutron wavelength of $\lambda_{i}=7 \AA$ yielded an accessible $q$-range of $0.6 \leq q \leq 1.6 \AA^{-1}$ at zero energy transfer and an instrumental resolution of about $90 \mu \mathrm{eV}$ (full width at half maximum). The static structure factor maximum of the $\mathrm{Au}_{81} \mathrm{Si}_{19}$ melt is at $2.7 \AA^{-1}$, well outside of the $q$-range investigated here. ${ }^{6}$ Coherent contributions to the total dynamic structure factor $S(q, \omega)$ comprise a central Rayleigh line, due to thermal diffusion, and a Brillouin doublet corresponding to acoustic modes. ${ }^{7}$ The former is very broad and appears as a flat background in the signal, while the latter is expected to be well separated from the quasielastic line in the energy transfer range accessed here. ${ }^{4,8-10}$ The measured spectra are thus almost entirely dominated by the incoherent scattering of the gold atoms $\left(\sigma_{\text {inc }}^{\mathrm{Au}}=0.43, \sigma_{\text {inc }}^{\mathrm{Si}}\right.$ $=0.004 \mathrm{~b}$ ) and reflect their self-motion on long time and length scales. ${ }^{11}$

The raw data were normalized to the measured intensity of a vanadium standard, corrected for sample self-shielding and interpolated to constant $q$ using the FRIDA- 1 software. ${ }^{12}$ In the range of zero energy transfer, the elastic scattering from the empty container is on the order of the quasielastic scattering from the melt. Thus, to avoid introducing unnecessary uncertainty into the data reduction by subtraction of the empty container signal, the measured $S^{*}(q, \omega)$ was modeled to include the container scattering as

$$
S^{*}(q, \omega)=A(q) \delta(\omega)+b(q)+[1-A(q)] \mathcal{F}(q, \omega) .
$$

The first term represents the elastic scattering from the container, $b(q)$ denotes a $q$-dependent background arising from short-time processes in the melt, ${ }^{9,13}$ and $\mathcal{F}(q, \omega)$ is a function that describes the quasielastic broadening due to atomic diffusion. During fitting, each spectrum was convolved with the resolution function. ${ }^{11}$

In the time domain, structural relaxation is directly accessed via the intermediate scattering function $S(q, t)$, obtained through a numerical Fourier transform of $S^{*}(q, \omega \geq 0)$ at each temperature. Here, the instrumental resolution is removed by dividing $S(q, t)$ by the Fourier transform of the 
resolution function. ${ }^{14}$ Normalizing to the value at $t=0$ yields the self-correlation function $\Phi(q, t)=S(q, t) / S(q, 0)$. Between 0 to $\simeq 1 \mathrm{ps}$, phonons and a fast relaxation process result in a rapid decay in atomic correlations from $\Phi(q, t)=1 .^{15}$ At longer times, the relaxation of gold atoms leads to the final decay of $\Phi(q, t)$-i.e., the structural $\alpha$-relaxation. The dynamics of glass-forming liquids is often described by the stretching in time of correlation functions, ${ }^{16,17}$ and we model the line shape of $\Phi(q, t)$ using a stretched exponential, or KohlrauschWilliams-Watts, ${ }^{18}$ function of the form ${ }^{19}$

$$
\Phi(q, t)=f_{q} \exp \left[-\left(t /\left\langle\tau_{q}\right\rangle\right)^{\beta_{q}}\right]+c,
$$

where $f_{q}$ is the Debye-Waller factor, $\left\langle\tau_{q}\right\rangle$ is a mean relaxation time, and $\beta_{q}$ is the stretching exponent. The value of $\left\langle\tau_{q}\right\rangle$ is obtained through $\left\langle\tau_{q}\right\rangle=\tau_{q} \beta_{q}^{-1} \Gamma\left(\beta_{q}^{-1}\right)$, where $\Gamma$ is the gamma function. The constant $c$ describes a flat background of around $15 \%$ in $\Phi(q, t)$, which arises from the elastic scattering of the niobium container and varies weakly with $q$ and temperature. In the energy domain, the quasielastic component $\mathcal{F}(q, \omega)$ is modeled by a numerical Fourier transform of the stretched exponential equation. The parameters $\left\langle\tau_{q}\right\rangle$ and $\beta_{q}$ are therefore also determined by fitting to Eq. (1).

Initially, $\beta_{q}$ was treated as a free parameter. At the four highest investigated temperatures, fitting in both energy and time domains consistently revealed a $q$-independent $\beta_{q} \simeq 1$ for $q$-values up to and including $1.0 \AA^{-1}$. Above $\sim 1 \AA^{-1}$, the decay of the $\alpha$-relaxation approaches the time scale of the aforementioned fast process, ${ }^{19}$ leading to a systematic decrease in $\beta_{q}$ with increasing $q$. Therefore, for further data analysis at these temperatures, a fixed value $\beta_{q}=1$ was used. At $713 \mathrm{~K}$, the data exhibit pronounced stretching over the entire accessible $q$-range with a mean $\beta_{q}=0.5$. The combined data analysis in both energy and time domains allows us to test for inconsistencies in the fitting results and thereby reduce systematic error.

Figure 1(a) shows the measured $S^{*}(q, \omega)$ of the $\mathrm{Au}_{81} \mathrm{Si}_{19}$ melt at 1103 and $713 \mathrm{~K}$, normalized to the

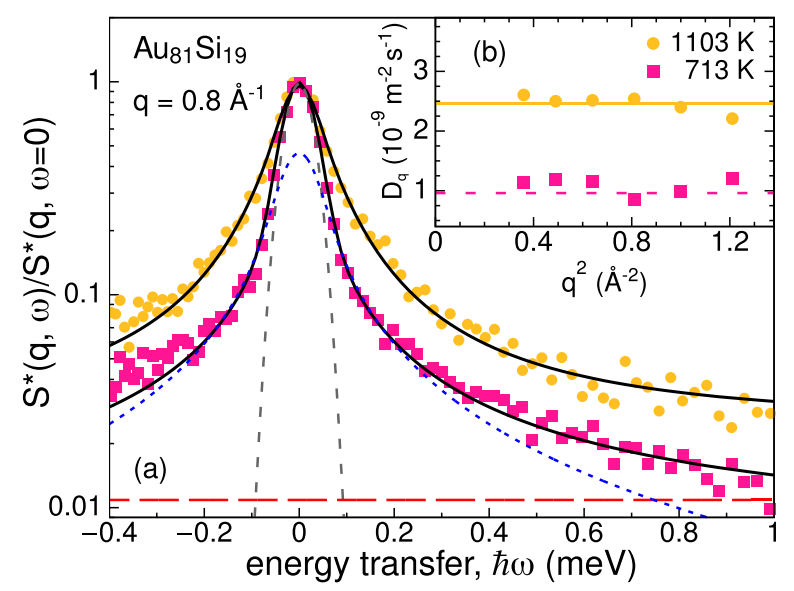

FIG. 1. (a) Measured $S^{*}(q, \omega)$ of the $\mathrm{Au}_{81} \mathrm{Si}_{19}$ melt at 1103 and $713 \mathrm{~K}$ along with fits to the model in Eq. (1) (solid lines). At $713 \mathrm{~K}$, the dashed, short dashed, and long dashed lines indicate the elastic, quasielastic, and background components of the total fit, respectively. (b) Rescaling of the fitted relaxation times as $D_{q}=\left(\left\langle\tau_{q}\right\rangle q^{2}\right)^{-1}$. The data are well described by a constant horizontal line in the limit $q \rightarrow 0$, giving the self-diffusion coefficient of gold on an absolute scale. Error bars are on the order of the symbol size. magnitude of the elastic peak. The spectra are well described by Eq. (1). In the hydrodynamic limit of $q \rightarrow 0$, the broadening of the quasielastic linewidth and hence the inverse of the relaxation time exhibit a characteristic dependence on $q^{2}$ [Fig. 1(b)]. By fitting to the equation $D=\left(\left\langle\tau_{q}\right\rangle q^{2}\right)^{-1}$, the self-diffusion coefficient $D$ of gold can thus be calculated on an absolute scale, given by horizontal lines in Fig. 1(b). ${ }^{7}$

In Fig. 2, the $D_{\mathrm{Au}}$ determined by QENS is shown as a function of inverse temperature. The values are averaged from both energy and time domain analyses. Both methods are proportional to $q^{2}$ within $10 \%$ or less. In the temperature range investigated, $D_{\mathrm{Au}}$ is well described by an Arrhenius law

$$
D_{\text {Au }}=D_{0} \exp \left(-E_{A} / k_{B} T\right)
$$

with an activation energy $E_{A}=(174 \pm 3) \mathrm{meV}$ atom $^{-1}$ and a $D_{0}=(16.9 \pm 3.3) \times 10^{-9} \mathrm{~m}^{2} \mathrm{~s}^{-1}$. The QENS results in Fig. 2 are shown together with those taken from gold radiotracer experiments using the capillary shear cell technique. ${ }^{20}$ In the range of the collected QENS data, both sets of diffusion coefficients show excellent agreement. Such good agreement with capillary measurements is rare, as their accuracy is often hampered by gravity-driven convective flow effects. ${ }^{13}$ Indeed, at higher temperatures, the shear cell data exhibit an anomalous change in slope, presumably a result of a convection-enhanced diffusion profile. Jakse et al. ${ }^{21}$ have determined a mean self-diffusivity in this melt using molecular dynamics (MD) simulations, which is in good agreement with experimental values.

At the $T_{m}$ of this alloy, we derive $D_{\mathrm{Au}}$ in $\mathrm{Au}_{81} \mathrm{Si}_{19}$ as $7.5( \pm 0.4) \times 10^{-10} \mathrm{~m}^{2} \mathrm{~s}^{-1}$, and $3.7( \pm 0.1) \times 10^{-9} \mathrm{~m}^{2} \mathrm{~s}^{-1}$ at the $T_{m}$ of pure liquid gold. The latter value is twice as large as that determined in MD simulations of pure gold, $2.0 \times 10^{-9} \mathrm{~m}^{2} \mathrm{~s}^{-1}, 22$ indicating that the addition of silicon actually speeds up the atomic mobility of gold in this melt. Essentially, this is the result of a less densely packed local structure, ${ }^{22}$ in which the more mobile silicon atoms reduce the effective energy barriers for atomic transport. Similar

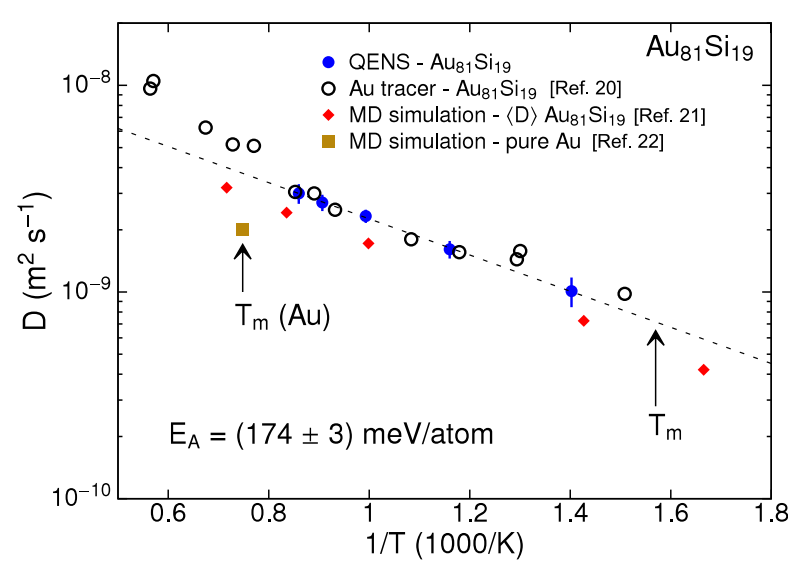

FIG. 2. Self-diffusion coefficient of gold in the $\mathrm{Au}_{81} \mathrm{Si}_{19}$ melt measured with QENS (filled circles) as a function of inverse temperature. The dashed line is a fit to an Arrhenius function. Values from gold radiotracer experiments (open circles) are also shown ${ }^{20}$ (error bars are on the order of the symbol size), along with mean self-diffusion coefficients $\langle D\rangle$ from MD simulations $^{21}$ (filled diamonds). The filled square is the self-diffusion coefficient of pure liquid gold from MD simulations. ${ }^{22}$ 
effects are observed in melts of gold-aluminum. ${ }^{23}$ The enhanced diffusivity of gold in the $\mathrm{Au}_{81} \mathrm{Si}_{19}$ melt also goes along with a low $E_{A}$, lower than what is found in melts of pure copper, nickel, titanium, ${ }^{4}$ and even aluminum $\left[E_{A}=(280 \pm 70) \mathrm{meV} \text { atom }^{-1}\right]^{8}$.

The class of fragile glass-forming liquids exhibits a strong slowing down of their dynamics during cooling ${ }^{24}$ and can be characterized by a low $E_{A}$ at high temperatures, similar to that reported here. A high fragility could be a boon to glass formation in $\mathrm{Au}_{81} \mathrm{Si}_{19}$, as it would imply an increasingly sluggish dynamics during cooling through the temperature range where nucleation and crystal growth become relevant. However, thermodynamic and structural aspects, such as a pronounced short-range ordering in the liquid versus the strong tendency for phase separation in the eutectic solid, should be taken into account as well. ${ }^{6,22}$ For example, glass formation is generally not found in the compositionally similar near-eutectic $\mathrm{Au}_{80} \mathrm{Al}_{20}$ melt-most likely the result of the preferential formation of stable intermetallic compounds. ${ }^{23,25}$

At high temperatures and for $0.6 \leq q \leq 1.0 \AA^{-1}$, a $q$-rescaling of $\Phi(q, t)$ as $\hat{\Phi}(q, \hat{t})=\left[\Phi\left(q, t /\left\langle\tau_{q}\right\rangle\right)-c\right] / f_{q}$ holds well with a mean $\beta_{q}=1$ [Fig. 3(a)]. Furthermore, in the temperature range of some $300 \mathrm{~K}$, a time-temperature-superposition (TTS) holds also with a simple exponential decay [Fig. 3(b)]. Thus, in this temperature and $q$-range, the selfmotion of gold atoms in the $\mathrm{Au}_{81} \mathrm{Si}_{19}$ melt can be described via Brownian diffusion, consistent with the predictions of hydrodynamics in the small $q$ limit. ${ }^{7,26}$ However, TTS is violated at $713 \mathrm{~K}$ by the pronounced stretching of $\Phi(q, t)$ [Fig. 3(b)] - an indication that the hydrodynamic regime is no longer reached on the spatial length scale probed here by the smallest accessible $q$; i.e., $2 \pi / q \approx 10 \AA$. Although structural relaxation at $713 \mathrm{~K}$ deviates significantly from the simple exponential predicted by hydrodynamic theory, the $\left\langle\tau_{q}\right\rangle$, nonetheless, obeys a $q^{2}$ proportionality, allowing us to extract an apparent self-diffusion coefficient commensurate with the QENS data at higher temperatures [Fig. 1(b)]. Thus,

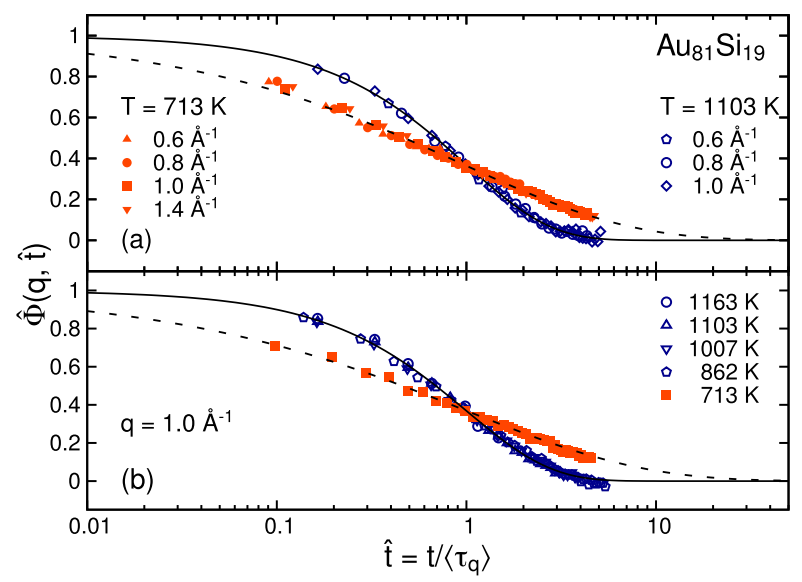

FIG. 3. (a) Rescaling in $q$ of $\Phi(q, t)$ at 1103 and $713 \mathrm{~K}$. At high temperatures, the data up to and including $q=1.0 \AA^{-1}$ are well described with a simple exponential decay (solid line, $\beta_{q}=1.0$ ), while at $713 \mathrm{~K}$, the line shape crosses over to a highly stretched exponential decay over all accessible $q$ (dashed line, $\beta_{q}=0.5$ ). (b) Time-temperature-superposition (TTS) of $\Phi(q, t)$ at $q=1.0 \AA^{-1}$. A simple exponential decay (solid line) holds over some $300 \mathrm{~K}$. The stretching at $713 \mathrm{~K}$ implies a violation of TTS. the $\left\langle\tau_{q}\right\rangle$ determined at this temperature appears to follow the same temperature and $q$-dependence as the $\tau$ from the hydrodynamic diffusion mode appearing in the limit $q \rightarrow 0$.

In general, the stretching of correlation functions seems to be more pronounced in fragile liquids and has been linked to the presence of spatial and dynamical heterogeneities in glass-forming ${ }^{27}$ and even simple liquids. ${ }^{28}$ At $713 \mathrm{~K}$, the stretching observed over the accessible $q$-range indicates a broad distribution of $\tau$, independent of the length scale probed, and suggests the onset of a heterogeneous dynamics. Furthermore, the crossover from diffusion-like to stretched exponential dynamics over a narrow temperature range would be consistent with a very fragile liquid although no dramatic slowing down is observed in the self-diffusion. This can be attributed to our aforementioned observation that, despite the large decrease in $\beta_{q}$ and consequent broadening in the relaxation time spectrum, the $\left\langle\tau_{q}\right\rangle$, nonetheless, follows a hydrodynamic $q^{2}$-scaling.

In Fig. 4(a), we compare the $D_{\mathrm{Au}}$ obtained here with the inverse of the $\alpha$-relaxation time $\tau_{\alpha}$, obtained from MD simulations of the self-intermediate scattering function. ${ }^{29}$ These two quantities agree very well within a proportionality constant and exhibit a comparable temperature dependence despite the emergence of a strong stretching dynamics at the lowest temperature. A similar comparison using the $\tau$ obtained at $q=1.6 \AA^{-1}$ in QENS is also shown in Fig. 4(a). The different proportionality constant is due simply to the larger $\tau$ at this $q$. For both sets of $\tau$, this proportionality holds over the entire investigated temperature range and can be alternatively represented by the relation $D \tau=$ const in Fig. $4(\mathrm{~b}) .{ }^{30}$ The horizontal lines represent the average value of $D \tau$.

It should be noted that the above relation holds even at $q=1.6 \AA^{-1}$, where the probed dynamics is likely to be a mixture of collective and self motion. Thus, taken together with the MD results, our experimental findings indicate that the self-diffusion of gold and collective relaxation times

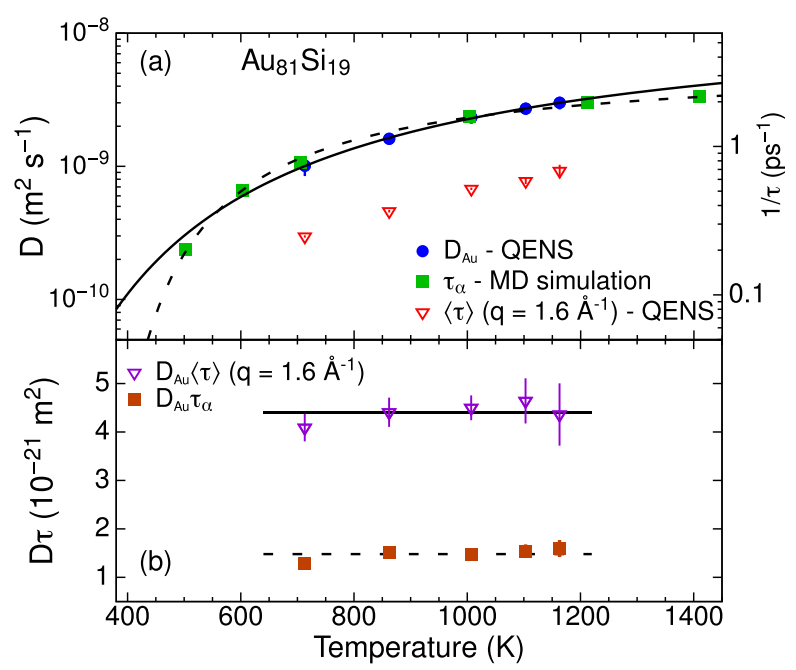

FIG. 4. (a) Temperature dependence of $D_{\text {Au }}$ (left axis) versus $1 / \tau$ (right axis). The vertical axes have been scaled to highlight the similar temperature dependences of $D_{\mathrm{Au}}$ and $\tau_{\alpha}$. The solid line is the Arrhenius fit of $D_{\mathrm{Au}}$, and the dashed line is a fit of $\tau_{\alpha}$ to the VFT equation. (b) The product $D \tau$ is the proportionality constant between self-diffusion and structural relaxation time at a given temperature. Missing values of $\tau_{\alpha}$ were interpolated according to the VFT fit. The horizontal lines represent the average value of $D \tau$. 
follow the same temperature dependence. Similar findings are observed in the $\mathrm{Zr}_{46.5} \mathrm{Ti}_{18.2} \mathrm{Cu}_{7.5} \mathrm{Ni}_{10} \mathrm{Be}_{27.5}$ melt and suggest a common time scale for structural relaxation, due to pronounced chemical short-range order and a large packing density. ${ }^{31}$

The temperature dependence of $\tau_{\alpha}$ can be well described by the empirical Vogel-Fulcher-Tammann (VFT) equation $\tau(T)=\tau_{0} \exp \left[D^{*} T_{0} /\left(T-T_{0}\right)\right]^{32} \quad$ Smaller $D^{*}$ values are characteristic of kinetically fragile liquids. ${ }^{24}$ As the fitted points are in the equilibrium liquid and therefore far away from $T_{0}$, we set $T_{0}$ equal to $T_{g}=291 \mathrm{~K}$, in order to avoid an over-parameterization of the data. ${ }^{33}$ The fitted values of $\tau_{0}=2.6 \times 10^{-13} \mathrm{~s}$ and $D^{*}=2.2$ indicate a very fragile liquid.

In conclusion, we investigated the microscopic dynamics in $\mathrm{Au}_{81} \mathrm{Si}_{19}$ over a range of $450 \mathrm{~K}$ in the equilibrium melt using QENS. The self-diffusivity of gold in this melt obeys an Arrhenius temperature dependence with a low $E_{A}$ despite its glass-forming nature. In violation of TTS, structural relaxation crosses over from a simple exponential at high temperatures to a highly stretched exponential present over all accessible $q$ at $713 \mathrm{~K}$. This reflects a rapid broadening in the relaxation time spectrum with decreasing temperature and suggests the onset of a heterogeneous dynamics, even in the equilibrium melt well above $T_{m}$. This behavior is generally consistent with that of a very fragile liquid. The observed phenomena are no doubt a result of alloying with silicon and warrant further examination with more detailed simulation and experimental efforts.

We thank Florian Kargl for many helpful discussions and Thomas Voigtmann for a critical reading of this manuscript. Wilfried Behr is gratefully acknowledged for the electron beam welding.

${ }^{1}$ Phase Transformations in Multicomponent Melts, edited by D. M. Herlach (Wiley-VCH Verlag GmbH \& Co KGaA, Weinheim, 2008).

${ }^{2}$ H. Okamoto and T. B. Massalski, Bull. Alloy Phase Diagrams 4, 362 (1983).

${ }^{3}$ J. B. Hannon, S. Kodambaka, F. M. Ross, and R. M. Tromp, Nature 440, 69 (2006); J. C. Mccloskey, S. Aithal, and P. R. Welch, Gold Bull. 34, 3 (2001); W. Klement, R. Willens, and P. Duwez, Nature 187, 896 (1960); J. Schroers, B. Lohwongwatana, W. L. Johnson, and A. Peker, Appl. Phys. Lett. 87, 061912 (2005).

${ }^{4}$ A. Meyer, EPJ Web Conf. 83, 01002 (2015).

${ }^{5}$ T. Unruh, J. Neuhaus, and W. Petry, Nucl. Instrum. Methods Phys. Res., Sect. A 580, 1414 (2007); W. Lohstroh and Z. Evenson, J. Large-Scale Res. Facil. 1, A15 (2015).
${ }^{6}$ P. Chirawatkul, A. Zeidler, P. Salmon, S. Takeda, Y. Kawakita, T. Usuki, and H. Fischer, Phys. Rev. B 83, 014203 (2011).

${ }^{7}$ J. P. Boon and S. Yip, Molecular Hydrodynamics (McGraw-Hill, New York, 1980).

${ }^{8}$ F. Kargl, H. Weis, T. Unruh, and A. Meyer, J. Phys.: Conf. Ser. 340, 012077 (2012).

${ }^{9}$ H. Weis, T. Unruh, and A. Meyer, High Temp.-High Press. 42, 39 (2013).

${ }^{10}$ E. Guarini, U. Bafile, F. Barocchi, A. De Francesco, E. Farhi, F. Formisano, A. Laloni, A. Orecchini, A. Polidori, M. Puglini, and F. Sacchetti, Phys. Rev. B 88, 104201 (2013).

${ }^{11}$ For gold and silicon, the coherent scattering lengths are $b_{c}=7.63$ and $b_{c}=4.15 \mathrm{fm}$, respectively. The weight of the concentration-fluctuation partial static structure factor $S_{c c}(q)$ is small enough, that we can neglect effects of inter-diffusion, arising from concentration fluctuations.

${ }^{12} \mathrm{See} \mathrm{http://sourceforge.net/projects/frida/} \mathrm{for} \mathrm{source} \mathrm{code.}$

${ }^{13}$ A. Meyer, Phys. Rev. B 81, 012102 (2010).

${ }^{14}$ C. C. Yuan, F. Yang, F. Kargl, D. Holland-Moritz, G. G. Simeoni, and A. Meyer, Phys. Rev. B 91, 214203 (2015).

${ }^{15}$ A. Meyer, J. Wuttke, W. Petry, O. Randl, and H. Schober, Phys. Rev. Lett. 80, 4454 (1998); A. Meyer, J. Wuttke, and W. Petry, J. Non-Cryst. Solids 250-252, 116 (1999).

${ }^{16}$ W. Götze and L. Sjögren, Rep. Prog. Phys. 55, 241 (1992).

${ }^{17}$ C. A. Angell, K. L. Ngai, G. B. McKenna, P. F. McMillan, and S. W. Martin, J. Appl. Phys. 88, 3113 (2000).

${ }^{18}$ R. Kohlrausch, Ann. Phys. Chem. 167, 179 (1854); G. Williams and C. Watts, Trans. Faraday Soc. 66, 80 (1970).

${ }^{19}$ F. Kargl, A. Meyer, M. Koza, and H. Schober, Phys. Rev. B 74, 014304 (2006).

${ }^{20}$ A. Bruson and M. Gerl, J. Appl. Phys. 53, 3616 (1982).

${ }^{21}$ N. Jakse, T. L. T. Nguyen, and A. Pasturel, J. Phys.: Condens. Matter 23, 404205 (2011).

${ }^{22}$ N. Jakse, T. L. T. Nguyen, and A. Pasturel, J. Chem. Phys. 137, 204504 (2012).

${ }^{23}$ H. L. Peng, T. Voigtmann, G. Kolland, H. Kobatake, and J. Brillo, Phys. Rev. B 92, 184201 (2015).

${ }^{24}$ C. A. Angell, Science 267, 1924 (1995).

${ }^{25}$ T. Egami, M. Ojha, D. M. Nicholson, D. V. Louzguine-Luzgin, N. Chen, and A. Inoue, Philos. Mag. 92, 655 (2012).

${ }^{26}$ F. Weysser, A. M. Puertas, M. Fuchs, and T. Voigtmann, Phys. Rev. E 82, 011504 (2010).

${ }^{27}$ R. Böhmer, K. L. Ngai, C. A. Angell, and D. J. Plazek, J. Chem. Phys. 99, 4201 (1993); M. D. Ediger, C. A. Angell, and S. R. Nagel, J. Phys. Chem. 100, 13200 (1996).

${ }^{28}$ F. Demmel and C. Morkel, Phys. Rev. E 85, 051204 (2012).

${ }^{29}$ A. Pasturel and N. Jakse, Phys. Rev. B 84, 134201 (2011).

${ }^{30}$ The relationship between $D$ and $1 / \tau$ can be described in both cases with a squared correlation coefficient of $r^{2} \sim 0.94$.

${ }^{31}$ F. Yang, T. Unruh, and A. Meyer, Europhys. Lett. 107, 26001 (2014); J. Brillo, A. Pommrich, and A. Meyer, Phys. Rev. Lett. 107, 165902 (2011); T. Voigtmann, A. Meyer, D. Holland-Moritz, S. Stüber, T. Hansen, and T. Unruh, Europhys. Lett. 82, 66001 (2008).

${ }^{32}$ H. Vogel, Phys. Z. 22, 645 (1921); G. Fulcher, J. Am. Ceram. Soc. 8, 339 (1925); G. Tammann and W. Hesse, Z. Anorg. Allg. Chem. 156, 245 (1926).

${ }^{33}$ H. S. Chen and D. Turnbull, J. Appl. Phys. 38, 3646 (1967). 